Research Article

\title{
General Randić index of unicyclic graphs with given number of pendant vertices
}

\author{
Tomáš Vetrík ${ }^{1, *}$, Selvaraj Balachandran ${ }^{2}$
}

${ }^{1}$ Department of Mathematics and Applied Mathematics, University of the Free State, Bloemfontein, South Africa

${ }^{2}$ Department of Mathematics, School of Arts, Sciences and Humanities, SASTRA Deemed University, Thanjavur, India

(Received: 1 December 2021. Received in revised form: 21 December 2021. Accepted: 25 December 2021. Published online: 4 January 2022.)

(C) 2022 the authors. This is an open access article under the CC BY (International 4.0) license (www.creativecommons.org/licenses/by/4.0/).

\begin{abstract}
For $a \in \mathbb{R}$ and a graph $G$, the general Randić index is defined as $R_{a}(G)=\sum_{u v \in E(G)}\left[d_{G}(u) d_{G}(v)\right]^{a}$, where $E(G)$ is the edge set of $G$, and $d_{G}(u)$ and $d_{G}(v)$ are degrees of the vertices $u$ and $v$ in $G$, respectively. For $-0.64 \leq a<0$, we give lower bounds on the general Randić index for unicyclic graphs with given number of pendant vertices, and with given order and number of pendant vertices. The extremal graphs are presented as well. Lower bounds on the classical Randić index are corollaries of our bounds on the general Randić index.
\end{abstract}

Keywords: general Randić index; unicyclic graph; pendant vertex; degree.

2020 Mathematics Subject Classification: 05C07, 05C09.

\section{Introduction}

Let $G$ be a simple connected graph with vertex set $V(G)$ and edge set $E(G)$. The order of $G$ is the number of vertices in $V(G)$. The degree $d_{G}(u)$ of a vertex $u \in V(G)$ is the number of edges incident with a vertex $u$. A pendant vertex is a vertex of degree one in $G$. A unicyclic graph is a connected graph with exactly one cycle. For $p \geq 3$ and $k \geq 0$, we denote by $C_{p, k}$ the unicyclic graph obtained from the cycle $C_{p}$ by joining one vertex of $C_{p}$ with $k$ new vertices.

In 1998, Bollobás and Erdős [2] introduced the general Randić index

$$
R_{a}(G)=\sum_{u v \in E(G)}\left[d_{G}(u) d_{G}(v)\right]^{a}
$$

of a graph $G$, where $a$ is any real number. For $a=-\frac{1}{2}$, we obtain the classical Randić index

$$
R_{-\frac{1}{2}}(G)=R(G)=\sum_{u v \in E(G)}\left[d_{G}(u) d_{G}(v)\right]^{-\frac{1}{2}} .
$$

The general Randić index belongs to well-known general topological indices. The $R_{a}$ index for unicyclic graphs with prescribed order has been studied in several papers. Wu and Zhang [12] found the unicyclic graphs with the smallest $R_{a}$ index for $a \geq-1$. For $a<-1$, unicyclic graphs with the smallest $R_{a}$ index were given by Li, Wang and Zhang [9]. The unicyclic graph having the largest $R_{a}$ index was given by Chen [3] for $-0.58<a<0$. For $a>0$, the same problem was studied by Li, Shi and Xu [7]. A survey on Randić indices was given by Li and Shi [6].

Alfuraidan et al. [1] found the graph with given order and diameter having the smallest $R_{a}$ index for $-0.64 \leq a<0$. Sharp bounds on the $R_{a}$ index for trees with given order and number of pendant vertices were given in [5], [8] and [10]. Related results for the general sum-connectivity index were presented for example in [4] and [11].

For $-0.64 \leq a<0$, we give lower bounds on the general Randić index for unicyclic graphs with given number of pendant vertices, and with given order and number of pendant vertices.

\section{Preliminary results}

Lemma 2.1 was given in [1] and it is used in the proofs of Lemmas 2.2 and 2.3.

Lemma 2.1. For $-1 \leq a<0$ and $x \geq 1$,

$$
\left(1+\frac{1}{x}\right)^{1-a}>1+\frac{1-a}{x} .
$$


Lemmas 2.2 and 2.3 are similar. It would be possible to present a more general result with Lemmas 2.2 and 2.3 being special cases of that result, but it would be a bit more complicated to follow its proof. Therefore, we present two separate lemmas.

Lemma 2.2. Let $-1 \leq a<0$ and $s \geq 2$. For $x \geq 2$, the function

$$
f_{1}(x)=\left(x-1+s^{a}\right) x^{a}-\left(x-2+s^{a}\right)(x-1)^{a}
$$

is strictly decreasing.

Proof. We obtain

$$
\begin{aligned}
f_{1}^{\prime}(x) & =x^{a}+\left(x-1+s^{a}\right) a x^{a-1}-(x-1)^{a}-\left(x-2+s^{a}\right) a(x-1)^{a-1} \\
& =\left[x+\left(x-1+s^{a}\right) a\right] x^{a-1}-\left[x-1+\left(x-2+s^{a}\right) a\right](x-1)^{a-1} .
\end{aligned}
$$

Let us prove that for every $x \geq 2$, we have $f_{1}^{\prime}(x)<0$.

Suppose to the contrary that for at least one value $x_{1} \in[2, \infty)$, we have $f_{1}^{\prime}\left(x_{1}\right) \geq 0$. Thus

$$
\left(x_{1}+a x_{1}-a+s^{a} a\right) x_{1}^{a-1} \geq\left(x_{1}+a x_{1}-2 a+s^{a} a-1\right)\left(x_{1}-1\right)^{a-1} .
$$

We have

$$
x_{1}+a x_{1}-2 a+s^{a} a-1=\left(x_{1}-1\right)(1+a)-a\left(1-s^{a}\right)>0,
$$

since $1+a \geq 0$ and $1-s^{a}>0$. Therefore, we get

$$
1+\frac{1+a}{x_{1}+a x_{1}-2 a+s^{a} a-1} \geq \frac{\left(x_{1}-1\right)^{a-1}}{x_{1}^{a-1}}=\left(1+\frac{1}{x_{1}-1}\right)^{1-a}>1+\frac{1-a}{x_{1}-1},
$$

where the strict inequality is obtained from Lemma 2.1. Thus

$$
\begin{aligned}
0 & >(1-a)\left(x_{1}+a x_{1}-2 a+s^{a} a-1\right)-(1+a)\left(x_{1}-1\right) \\
& =-a\left[(1+a)\left(x_{1}+s^{a}\right)-2\left(s^{a}+a\right)\right] \\
& >-a\left[(1+a)\left(x_{1}+s^{a}\right)-2(1+a)\right] \\
& =-a(1+a)\left(x_{1}-2+s^{a}\right) .
\end{aligned}
$$

Since $x_{1} \geq 2$ and $0<s^{a}<1$, we obtain $x_{1}+s^{a}-2>0$. Then

$$
-a(1+a)\left(x_{1}-2+s^{a}\right) \geq 0,
$$

which is a contradiction. Thus for $x \geq 2$, we have $f_{1}^{\prime}(x)<0$. Hence $f_{1}(x)$ is strictly decreasing.

Rather than saying that the proof of Lemma 2.3 is similar to the proof of Lemma 2.2, we present a complete proof of Lemma 2.3 as well.

Lemma 2.3. Let $-1 \leq a<0, s_{1} \geq 2$ and $s_{2} \geq 2$. For $x \geq 3$, the function

$$
f_{2}(x)=\left(x-2+s_{1}^{a}+s_{2}^{a}\right) x^{a}-\left(x-3+s_{1}^{a}+s_{2}^{a}\right)(x-1)^{a}
$$

is strictly decreasing.

Proof. We obtain

$$
\begin{aligned}
f_{2}^{\prime}(x) & =x^{a}+\left(x-2+s_{1}^{a}+s_{2}^{a}\right) a x^{a-1}-(x-1)^{a}-\left(x-3+s_{1}^{a}+s_{2}^{a}\right) a(x-1)^{a-1} \\
& =\left[x+\left(x-2+s_{1}^{a}+s_{2}^{a}\right) a\right] x^{a-1}-\left[x-1+\left(x-3+s_{1}^{a}+s_{2}^{a}\right) a\right](x-1)^{a-1} .
\end{aligned}
$$

Let us prove that for every $x \geq 3$, we have $f_{2}^{\prime}(x)<0$.

Suppose to the contrary that for at least one value $x_{1} \in[3, \infty)$, we have $f_{2}^{\prime}\left(x_{1}\right) \geq 0$. Thus

$$
\left(x_{1}+a x_{1}-2 a+s_{1}^{a} a+s_{2}^{a} a\right) x_{1}^{a-1} \geq\left(x_{1}+a x_{1}-3 a+s_{1}^{a} a+s_{2}^{a} a-1\right)\left(x_{1}-1\right)^{a-1} .
$$

We have

$$
x_{1}+a x_{1}-3 a+s_{1}^{a} a+s_{2}^{a} a-1=\left(x_{1}-1\right)(1+a)-a\left(2-s_{1}^{a}-s_{2}^{a}\right)>0,
$$


since $1+a \geq 0$ and $2-s_{1}^{a}-s_{2}^{a}>0$. Therefore, we get

$$
1+\frac{1+a}{x_{1}+a x_{1}-3 a+s_{1}^{a} a+s_{2}^{a} a-1} \geq \frac{\left(x_{1}-1\right)^{a-1}}{x_{1}^{a-1}}=\left(1+\frac{1}{x_{1}-1}\right)^{1-a}>1+\frac{1-a}{x_{1}-1},
$$

where the strict inequality is obtained from Lemma 2.1. Thus

$$
\begin{aligned}
0 & >(1-a)\left(x_{1}+a x_{1}-3 a+s_{1}^{a} a+s_{2}^{a} a-1\right)-(1+a)\left(x_{1}-1\right) \\
& =-a\left[(1+a)\left(x_{1}-3+s_{1}^{a}+s_{2}^{a}\right)+2\left(1-s_{1}^{a}\right)+2\left(1-s_{2}^{a}\right)\right] \\
& >-a(1+a)\left(x_{1}-3+s_{1}^{a}+s_{2}^{a}\right)
\end{aligned}
$$

Since $x_{1} \geq 3,0<s_{1}^{a}<1$ and $0<s_{2}^{a}<1$, we obtain $x_{1}-3+s_{1}^{a}+s_{2}^{a}>0$. Then

$$
-a(1+a)\left(x_{1}-3+s_{1}^{a}+s_{2}^{a}\right) \geq 0,
$$

which is a contradiction. Thus for $x \geq 3$, we have $f_{2}^{\prime}(x)<0$. Hence $f_{2}(x)$ is strictly decreasing.

Lemmas 2.4 and 2.5 were presented in [1]. Lemmas 2.2, 2.3, 2.4 and 2.5 are used in the proof of Theorem 3.1.

Lemma 2.4. Let $-1 \leq a<0$ and $c \geq 2$. For $x \geq 1$, the function

$$
f_{3}(x)=(c x)^{a}-[(c-1) x]^{a}
$$

is strictly increasing.

Lemma 2.5 was presented in [1] for $x \geq 2$. However, the proof given in [1] holds for any $x>0$. Thus we state Lemma 2.5 for $x>0$. This lemma is used in the proof of Theorem 3.1 to compare $f_{4}(k)$ and $f_{4}(k+1)$ for $k \geq 1$.

Lemma 2.5. Let $-0.64 \leq a<0$. For $x \geq 2$, the function

$$
f_{4}(x)=\left(x-1+2^{a+1}\right)(x+1)^{a}-\left(x-1+2^{a}\right) x^{a}
$$

is strictly decreasing.

\section{Main result}

We present a lower bound on the general Randić index for unicyclic graphs with $n$ vertices and $k$ pendant vertices. The extremal graph is presented in Figure 1. Note that for every unicyclic graph, we have $0 \leq k \leq n-3$.

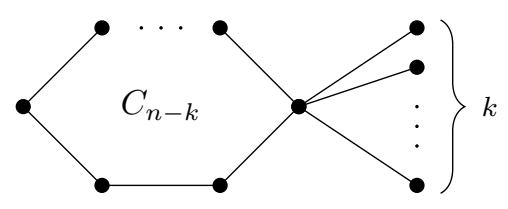

Figure 1: The graph $C_{n-k, k}$.

Theorem 3.1. Let $-0.64 \leq a<0$. For any unicyclic graph $G$ with $n$ vertices and $k$ pendant vertices, we have

$$
R_{a}(G) \geq(n-k-2) 4^{a}+\left(k+2^{a+1}\right)(k+2)^{a}
$$

with equality if and only if $G$ is $C_{n-k, k}$.

Proof. Clearly, $k \geq 0$ and $n \geq k+3$ for every unicyclic graph with given $n$ and $k$. For the graph $C_{n-k, k}$, we have

$$
\begin{aligned}
R_{a}(G) & =(n-k-2) 4^{a}+k(k+2)^{a}+2[2(k+2)]^{a} \\
& =(n-k-2) 4^{a}+\left(k+2^{a+1}\right)(k+2)^{a} .
\end{aligned}
$$

We prove the result by induction on $n$. The only unicyclic graph with $k=0$ pendant vertices and $n$ vertices is $C_{n}=C_{n, 0}$. For $n=k+3$, the only unicyclic graph with $k$ pendant vertices and $k+3$ vertices is $C_{3, k}$. So the result holds for those cases.

Let $n \geq k+4$ and $k \geq 1$. Assume that the result holds for all unicyclic graphs with at most $n-1$ vertices. Let $G$ be a unicyclic graph with $n$ vertices and $k$ pendant vertices. We denote the unique cycle in $G$ by $C$. Let $v$ be a pendant vertex 
furthest from $C$ in $G$ and let $G^{\prime}=G-v$. We denote the unique neighbor of $v$ in $G$ by $w$. Let $d_{G}(w)=p$. We distinguish the following cases.

Case 1: $w \in V(C)$.

Then $w$ is adjacent to two vertices $w_{1}, w_{2} \in V(C)$ and $p-2$ pendant vertices in $G$, where $3 \leq p \leq k+2$. We have $d_{G}\left(w_{1}\right)=s_{1} \geq 2$ and $d_{G}\left(w_{2}\right)=s_{2} \geq 2$. Then

$$
\begin{aligned}
R_{a}(G) & =R_{a}\left(G^{\prime}\right)+(p-2) p^{a}-(p-3)(p-1)^{a}+\left(s_{1} p\right)^{a}-\left[s_{1}(p-1)\right]^{a}+\left(s_{2} p\right)^{a}-\left[s_{2}(p-1)\right]^{a} \\
& =R_{a}\left(G^{\prime}\right)+\left(p-2+s_{1}^{a}+s_{2}^{a}\right) p^{a}-\left(p-3+s_{1}^{a}+s_{2}^{a}\right)(p-1)^{a} .
\end{aligned}
$$

Since $p \leq k+2$, by Lemma 2.3, we have

$$
\begin{aligned}
f_{2}(p) & =\left(p-2+s_{1}^{a}+s_{2}^{a}\right) p^{a}-\left(p-3+s_{1}^{a}+s_{2}^{a}\right)(p-1)^{a} \\
& \geq\left(k+s_{1}^{a}+s_{2}^{a}\right)(k+2)^{a}-\left(k-1+s_{1}^{a}+s_{2}^{a}\right)(k+1)^{a} \\
& =f_{2}(k+2),
\end{aligned}
$$

with equality if and only if $p=k+2$ (which means that $w$ is adjacent to $k$ pendant vertices in $G$ ). By Lemma 2.4 , for $s_{1}, s_{2} \geq 2$,

$$
f_{3}\left(s_{1}\right)=(k+2)^{a} s_{1}^{a}-(k+1)^{a} s_{1}^{a} \geq(k+2)^{a} 2^{a}-(k+1)^{a} 2^{a}=f_{3}(2)
$$

and

$$
f_{3}\left(s_{2}\right)=(k+2)^{a} s_{2}^{a}-(k+1)^{a} s_{2}^{a} \geq(k+2)^{a} 2^{a}-(k+1)^{a} 2^{a}=f_{3}(2)
$$

with equalities if and only if $s_{1}=2$ and $s_{2}=2$, respectively. Therefore

$$
R_{a}(G) \geq R_{a}\left(G^{\prime}\right)+\left(k+2^{a+1}\right)(k+2)^{a}-\left(k-1+2^{a+1}\right)(k+1)^{a} .
$$

Note that $G^{\prime}$ has $n-1$ vertices and $k-1$ pendant vertices. By the induction hypothesis,

$$
R_{a}\left(G^{\prime}\right) \geq(n-k-2) 4^{a}+\left(k-1+2^{a+1}\right)(k+1)^{a}
$$

with equality if and only if $G^{\prime}$ is $C_{n-k, k-1}$. Therefore

$$
R_{a}(G) \geq(n-k-2) 4^{a}+\left(k+2^{a+1}\right)(k+2)^{a}
$$

with equality if and only if $G$ is $C_{n-k, k}$.

Case 2: $w \notin V(C)$.

In this case, we prove that $R_{a}(G)>R_{a}\left(C_{n-k, k}\right)$. Since $v$ is a pendant vertex furthest from $C$ in $G$, there is exactly one non-pendant vertex adjacent to $w$ in $G$. We denote it by $w^{\prime}$. We have $d_{G}\left(w^{\prime}\right)=s \geq 2$. The other $p-1$ neighbors of $w$ in $G$ are pendant vertices. Note that $2 \leq p \leq k+1$.

Case 2.1: $p \geq 3$.

We have

$$
\begin{aligned}
R_{a}(G) & =R_{a}\left(G^{\prime}\right)+(p-1) p^{a}-(p-2)(p-1)^{a}+(s p)^{a}-[s(p-1)]^{a} \\
& =R_{a}\left(G^{\prime}\right)+\left(p-1+s^{a}\right) p^{a}-\left(p-2+s^{a}\right)(p-1)^{a} .
\end{aligned}
$$

Since $p \leq k+1$, by Lemma 2.2 , we have

$$
\begin{aligned}
f_{1}(p) & =\left(p-1+s^{a}\right) p^{a}-\left(p-2+s^{a}\right)(p-1)^{a} \\
& \geq\left(k+s^{a}\right)(k+1)^{a}-\left(k-1+s^{a}\right) k^{a} \\
& =f_{1}(k+1) .
\end{aligned}
$$

with equality if and only if $p=k+1$ (which means that $w$ is adjacent to $k$ pendant vertices in $G$ ). By Lemma 2.4, for $s \geq 2$,

$$
f_{3}(s)=(k+1)^{a} s^{a}-k^{a} s^{a} \geq(k+1)^{a} 2^{a}-k^{a} 2^{a}=f_{3}(2)
$$

with equality if and only if $s=2$. Therefore

$$
R_{a}(G) \geq R_{a}\left(G^{\prime}\right)+\left(k+2^{a}\right)(k+1)^{a}-\left(k-1+2^{a}\right) k^{a} .
$$


Note that $G^{\prime}$ has $n-1$ vertices and $k-1$ pendant vertices. By the induction hypothesis,

$$
R_{a}\left(G^{\prime}\right) \geq(n-k-2) 4^{a}+\left(k-1+2^{a+1}\right)(k+1)^{a} .
$$

Therefore

$$
\begin{aligned}
R_{a}(G) & \geq(n-k-2) 4^{a}+\left(k-1+2^{a+1}\right)(k+1)^{a}+\left(k+2^{a}\right)(k+1)^{a}-\left(k-1+2^{a}\right) k^{a} \\
& >(n-k-2) 4^{a}+\left(k+2^{a+1}\right)(k+2)^{a} \\
& =R_{a}\left(C_{n-k, k}\right),
\end{aligned}
$$

since by Lemma 2.5 ,

$$
\begin{aligned}
f_{4}(k) & =\left(k-1+2^{a+1}\right)(k+1)^{a}-\left(k-1+2^{a}\right) k^{a} \\
& >\left(k+2^{a+1}\right)(k+2)^{a}-\left(k+2^{a}\right)(k+1)^{a} \\
& =f_{4}(k+1) .
\end{aligned}
$$

Case 2.2: $p=2$.

Since $G^{\prime}$ has $n-1$ vertices and $k$ pendant vertices, by the induction hypothesis, we get

$$
R_{a}\left(G^{\prime}\right) \geq(n-k-3) 4^{a}+\left(k+2^{a+1}\right)(k+2)^{a}
$$

with equality if and only if $G^{\prime}$ is $C_{n-k-1, k}$. Using $c=2$ in Lemma 2.4, for $s \geq 2$, we obtain

$$
f_{3}(s)=(2 s)^{a}-s^{a} \geq 4^{a}-2^{a}=f_{3}(2)
$$

with equality if and only if $s=2$. Therefore

$$
\begin{aligned}
R_{a}(G) & =R_{a}\left(G^{\prime}\right)+2^{a}+(2 s)^{a}-s^{a} \\
& \geq(n-k-3) 4^{a}+\left(k+2^{a+1}\right)(k+2)^{a}+2^{a}+4^{a}-2^{a} \\
& =(n-k-2) 4^{a}+\left(k+2^{a+1}\right)(k+2)^{a}
\end{aligned}
$$

with equality if and only if $G^{\prime}$ is $C_{n-k-1, k}$ and $s=2$, which means that $G$ contains an edge $w w^{\prime}$ not in $C$ with $d_{G}(w)=$ $d_{G}\left(w^{\prime}\right)=2$. That is not possible, hence

$$
R_{a}(G)>(n-k-2) 4^{a}+\left(k+2^{a+1}\right)(k+2)^{a} .
$$

The proof is complete.

\section{Corollaries}

In this section, we give three corollaries. First, we present a lower bound on the $R_{a}$ index for unicyclic graphs only with given number of pendant vertices. The extremal graph is presented in Figure 2.

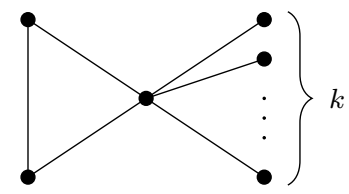

Figure 2: The graph $C_{3, k}$.

Corollary 4.1. Let $-0.64 \leq a<0$. For any unicyclic graph $G$ with $k$ pendant vertices, we have

$$
R_{a}(G) \geq\left(k+2^{a+1}\right)(k+2)^{a}+2^{2 a}
$$

with equality if and only if $G$ is $C_{3, k}$. 
Proof. By Theorem 3.1, for any unicyclic graph $G$ with $n$ vertices and $k$ pendant vertices, where $0 \leq k \leq n-3$, we have

$$
R_{a}(G) \geq(n-k-2) 4^{a}+\left(k+2^{a+1}\right)(k+2)^{a}
$$

with equality if and only if $G$ is $C_{n-k, k}$. The function

$$
f(n)=(n-k-2) 4^{a}+\left(k+2^{a+1}\right)(k+2)^{a}
$$

is strictly increasing, since $f^{\prime}(n)=4^{a}>0$. Thus, for $n \geq k+3$, we have $f(n) \geq f(k+3)$ with equality if and only if $n=k+3$. Therefore, for any unicyclic graph $G$ with $k$ pendant vertices,

$$
R_{a}(G) \geq f(k+3)=\left(k+2^{a+1}\right)(k+2)^{a}+2^{2 a}
$$

with equality if and only if $G$ is $C_{3, k}$.

We use $a=-\frac{1}{2}$ in Theorem 3.1 and Corollary 4.1 to obtain lower bounds on the classical Randić index.

Corollary 4.2. For any unicyclic graph $G$ with $n$ vertices and $k$ pendant vertices, we have

$$
R(G) \geq \frac{n-k}{2}+\frac{k+\sqrt{2}}{\sqrt{k+2}}-1
$$

with equality if and only if $G$ is $C_{n-k, k}$.

Corollary 4.3. For any unicyclic graph $G$ with $k$ pendant vertices, we have

$$
R(G) \geq \frac{k+\sqrt{2}}{\sqrt{k+2}}+\frac{1}{2}
$$

with equality if and only if $G$ is $C_{3, k}$.

\section{Acknowledgment}

The work of T. Vetrík is based on the research supported by the National Research Foundation of South Africa (Grant Number 129252).

\section{References}

[1] M. R. Alfuraidan, K. C. Das, T. Vetrík, S. Balachandran, General Randić index of unicyclic graphs with given diameter, Discrete Appl. Math. 306 (2022) 7-16.

[2] B. Bollobás, P. Erdős, Graphs of extremal weights, Ars Combin. 50 (1998) 225-233.

[3] D. Chen, Study of unicyclic graph with maximal general Randić index $R_{\alpha}$ for $\alpha<0$, Commun. Comput. Inf. Sci. 134 (2011) $136-141$.

[4] Q. Cui, L. Zhong, On the general sum-connectivity index of trees with given number of pendent vertices, Discrete Appl. Math. 222 (2017) $213-221$.

[5] Q. Cui, L. Zhong, The general Randić index of trees with given number of pendent vertices, Appl. Math. Comput. 302 (2017) 111-121.

[6] X. Li, Y. Shi, A survey on the Randić index, MATCH Commun. Math. Comput. Chem. 59 (2008) 127-156.

[7] X. Li, Y. Shi, T. Xu, Unicyclic graphs with maximum general Randić index for $\alpha>0$, MATCH Commun. Math. Comput. Chem. 56 (2006) 557-570.

[8] X. Li, Y. Shi, L. Zhong, Minimum general Randić index on chemical trees with given order and number of pendent vertices, MATCH Commun. Math. Comput. Chem. 60 (2008) 539-554.

[9] X. Li, L. Wang, Y. Zhang, Complete solution for unicyclic graphs with minimum general Randić index, MATCH Commun. Math. Comput. Chem. 55 (2006) 391-408.

[10] H. Liu, M. Lu, F. Tian, Trees of extremal connectivity index, Discrete Appl. Math. 154 (2006) 106-119.

[11] I. Tomescu, M. Arshad, On the general sum-connectivity index of connected unicyclic graphs with $k$ pendant vertices, Discrete Appl. Math. 181 (2015) 306-309.

[12] B. Wu, L. Zhang, Unicyclic graphs with minimum general Randić index, MATCH Commun. Math. Comput. Chem. 54 (2005) $455-464$. 